\title{
Analysis of Entropy Generation in a Rectangular Porous Duct
}

\author{
Shyam Lal Yadav, Ashok Kumar Singh \\ Department of Mathematics, Institute of Science, Banaras Hindu University, Varanasi, India \\ Email: slyadav4884@gmail.com, ashok@bhu.ac.in
}

Received 3 May 2016; accepted 25 July 2016; published 28 July 2016

Copyright (C) 2016 by authors and Scientific Research Publishing Inc.

This work is licensed under the Creative Commons Attribution International License (CC BY). http://creativecommons.org/licenses/by/4.0/

(c) (i) Open Access

\begin{abstract}
In this paper, we have considered a fully developed flow of a viscous incompressible fluid in a rectangular porous duct saturated with the same fluid. The duct is heated from the bottom for forced and mixed convection. The Brinkman model is used to simulate the momentum transfer in the porous duct. Using the momentum and thermal energy equations, the entropy generation has been obtained due to the heat transfer, viscous and Darcy dissipations. It is found from the mathematical analysis that the entropy generation is double when the viscous as well as the Darcy dissipations terms are taken in the thermal energy equation in comparison when the viscous as well as the Darcy dissipations terms are not taken in the thermal energy equation. This result clearly shows that there is no need of taking the viscous and Darcy dissipations terms in the thermal energy equation to obtain the entropy generation.
\end{abstract}

\section{Keywords}

Brinkman Model, Heat Flux, Fully Developed Flow, Viscosity Ratio, Entropy Generation

\section{Introduction}

The study of transport phenomena in the channel filled with saturated porous media has attracted considerable attention of scientists, engineers and experimentalists in present time. This attention is mainly due to the applications of this phenomenon in the field of electronics cooling system, geothermal system, storage of nuclear waste materials, microelectronics heat transfer equipment, coal and grain storage, crude oil production, catalytic converters, ground water pollution, fiber and granular insulations, solidification of castings, etc. The advancement in the thermal systems as well as the energy utilization during the convection in any fluid is one of the fundamental problems of the technological processes, because the improved thermal systems will provide better material processing, energy conservation and environmental effects. Also, because of applications to the cooling of electronic equipment there has been an increased interest in the forced convections in the channels and ducts 
filled with the porous media. One of the important viscous fluid flow situations in the porous media is the Poiseuille flow in a rectangular duct. In addition, convection through the porous medium may be found in the fiber and granular insulation, including structures for high power density, electric machines and nuclear reactors.

Viscosity is the measure of a fluid's resistance to the flow and it describes the internal friction of a moving fluid. Due to the added resistance of the porous structure, the effective viscosity $\mu_{e}$ of the porous medium is generally found different from the fluid viscosity $\mu$ and it depends on the properties of porous media (e.g., porosity and permeability). The effective viscosity of the porous matrix is generally larger than the fluid viscosity, however, the available literatures, unfortunately, exhibit a remarkable disagreement with respect to the viscosity ratio $\mu_{e} / \mu$. Brinkman [1] has taken both viscosities as same. Lundgren [2] has shown that, for the flow phenomenon near a random bed of spheres, the effective viscosity is larger than the fluid viscosity for high porosity and smaller for low porosity. Further, the viscosity ratio $\mu_{e} / \mu$ has been taken as greater than one in the studies of Adler and Mills [3] and Kim and Russell [4] while in Koplik, J., Levine, H., Zee, A. [5], Kolodziej [6], Nield and Bejan [7] and Breugem [8], it has been taken as less than one.

In addition to the analysis based on the basic conservation laws, the analysis of second-law of thermodynamics is important in understanding the entropy generation, which is attributed to the thermodynamic irreversibility. This kind of thermodynamic analysis is significant for studying the optimum operating conditions, which helps in designing a system with less entropy and destruction of available work (energy). According to the Gouy-Stodola theorem, the lost available work is directly proportional to the entropy generation. The utilization of the second law of thermodynamics in convective heat transfer is very well presented by Bejan [9] [10]. By comparing the results of various cross sectional duct geometries, Sahin [11] has shown that the circular duct geometry is favourable one when the frictional contribution of the entropy generation becomes dominant. Narusawa [12] has presented the theoretical and numerical studies to the cross-sectional rate of the entropy generation for a fully developed flow, consisting of contributions from heat transfer and viscous dissipation. By applying the first and second laws of thermodynamics to forced convection in a cylindrical annular space with isoflux boundary conditions, Mahmud and Fraser [13] have obtained the general expressions for the velocity and temperature distributions, entropy generation number and Bejan number. Mohammad [14] has demonstrated the flow field and heat transfer with laminar forced convection in conduits field with a porous material to different degrees. He calculated that the effect of the Darcy number on the heat transfer in a fully developed flow region may largely be neglected for $D a>1$ in the case of homogeneously filled channel.

Sauoli and Sauoli [15] have obtained the effect of the second law on laminar falling liquid film along an inclined heated plate by considering the upper surface of the liquid film free and adiabatic and the lower wall having constant heat flux. Their results show that the entropy generation increases for all values of the group parameters. Makinde and Osalusi [16] have studied the impact of the second law in the case of laminar flow in a channel field with saturated porous media. Yadav and Singh [17] have shown that the Darcy dissipation dominates the viscous dissipation everywhere of the channel except near the walls in a fully developed horizontal porous channel and further Yadav and Singh [18] have presented in details the analysis of entropy generation in an annular porous duct.

Heat transfer from solid walls to flowing fluids is an area of extreme scientific interest as well as of immense practical importance. Certain flows, passing through the bodies with high porosity, do not follow the Darcy's law and Brinkman's model is applicable for that type of flows. Neale and Nader [19] have also given the practical significance of the Brinkman extension of Darcy law. Accurate description of fluid flow behavior in the porous media is essential for successful design and operation of systems in this area. In the present paper, our main focus is to see the effect of viscous and Darcy dissipations in the energy equation of rectangular porous duct by employing the Brinkman model to calculate the non-dimensional entropy generation.

\section{Mathematical Formulation}

Consider the steady, laminar, two dimensional incompressible fluid flow in a saturated porous medium bounded by a rectangular duct of width, $2 \mathrm{~W}$ and height, $H$ with the origin of a coordinate system located at the corner of the rectangular duct as sketched in Figure 1, and the direction of gravity, $g$, in the negative $y$ direction. The temperature at the bottom and the top are kept at constant temperatures $T_{\text {bot }}$ and $T_{\text {top }}\left(<T_{\text {bot }}\right)$, respectively with small temperature difference $\Delta T=T_{\text {bot }}-T_{\text {top }}$; while the side boundaries are taken as adiabatic. For steady, low-Rayleigh number mixed convection, pressure variation may be decomposed into two parts, a variation in the 


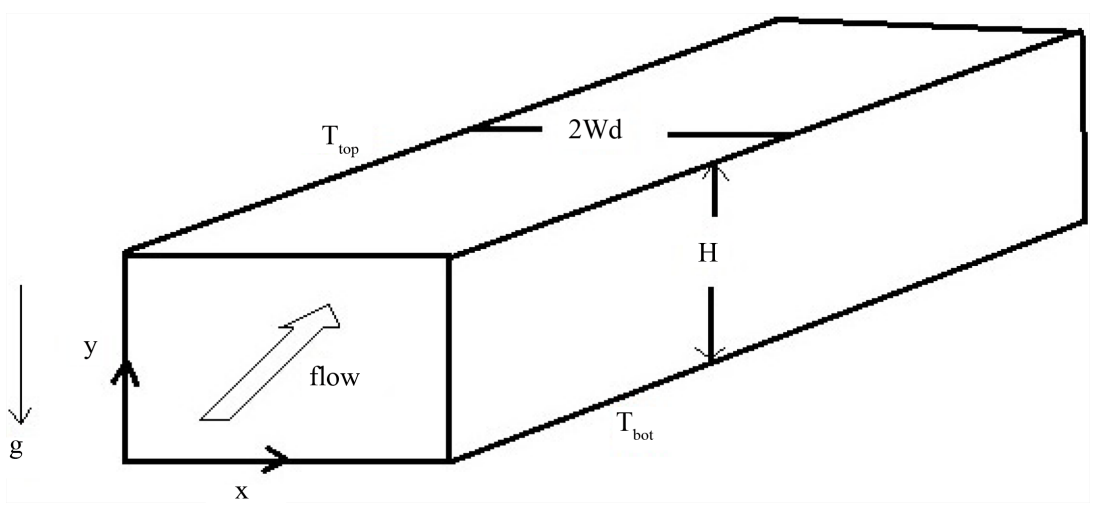

Figure 1. Schematic Diagram.

main flow $(z)$ direction and a cross sectional variation in the $x-y$ plane; i.e. $p=\bar{p}(z)+p^{\prime}(x, y, z)$ with $\mathrm{d} \bar{p} / \mathrm{d} z \gg \partial p^{\prime} / \partial x, \partial p^{\prime} / \partial y, \partial p^{\prime} / \partial z$.

We also assume that the fluid motion can adequately be described by Boussinesq approximation. Under the assumptions of constant thermo-physical properties and linear Boussinesq approximations, the governing conservation equations, namely the equation of continuity, momentum and thermal energy for the isotropic and homogeneous porous medium may be written as:

$$
\begin{gathered}
\frac{\partial u}{\partial x}+\frac{\partial v}{\partial y}+\frac{\partial w}{\partial z}=0 \\
\rho\left(u \frac{\partial u}{\partial x}+v \frac{\partial u}{\partial y}+w \frac{\partial u}{\partial z}\right)=-\frac{\partial p^{\prime}}{\partial x}+\mu_{e}\left(\frac{\partial^{2} u}{\partial x^{2}}+\frac{\partial^{2} u}{\partial y^{2}}+\frac{\partial^{2} u}{\partial z^{2}}\right)-\frac{\mu}{K} u, \\
\rho\left(u \frac{\partial v}{\partial x}+v \frac{\partial v}{\partial y}+w \frac{\partial v}{\partial z}\right)=-\frac{\partial p^{\prime}}{\partial y}+\mu_{e}\left(\frac{\partial^{2} v}{\partial x^{2}}+\frac{\partial^{2} v}{\partial y^{2}}+\frac{\partial^{2} v}{\partial z^{2}}\right)-\frac{\mu}{K} v+\rho g \beta\left(T-T_{R}\right), \\
\rho\left(u \frac{\partial w}{\partial x}+v \frac{\partial w}{\partial y}+w \frac{\partial w}{\partial z}\right)=-\frac{\partial \bar{p}}{\partial z}+\mu_{e}\left(\frac{\partial^{2} w}{\partial x^{2}}+\frac{\partial^{2} w}{\partial y^{2}}+\frac{\partial^{2} w}{\partial z^{2}}\right)-\frac{\mu}{K} w, \\
\rho c_{p}\left(u \frac{\partial T}{\partial x}+v \frac{\partial T}{\partial y}+w \frac{\partial T}{\partial z}\right)=k\left(\frac{\partial^{2} T}{\partial x^{2}}+\frac{\partial^{2} T}{\partial y^{2}}+\frac{\partial^{2} T}{\partial z^{2}}\right),
\end{gathered}
$$

For the fully developed flow $(\partial \vec{V} / \partial z=\partial T / \partial z=0)$, the above equations may be observed that, these are identical to the governing equations of the Rayleigh-Benard convection in the porous medium (i.e. natural convection in rectangular porous cavity). The entropy is generated due to the presence of irreversibility, and entropy generation is adopted as a quantative measure of the irreversibility associated with a thermal process. Therefore, under supercritical conditions the longitudinal convective rolls are superposed to the forced convective flow. The local volumetric rate of entropy generation, $\dot{S}_{G}^{\prime \prime \prime}\left[\mathrm{W} / \mathrm{m}^{3} \cdot \mathrm{K}\right]$ based on second law of thermodynamics, is given as

$$
\begin{aligned}
\dot{S}_{G}^{\prime \prime \prime}= & \frac{k}{T^{2}}\left[\left(\frac{\partial T}{\partial x}\right)^{2}+\left(\frac{\partial T}{\partial y}\right)^{2}+\left(\frac{\partial T}{\partial z}\right)^{2}\right]+\frac{\mu_{e}}{T}\left[2\left(\left(\frac{\partial u}{\partial x}\right)^{2}+\left(\frac{\partial v}{\partial y}\right)^{2}+\left(\frac{\partial w}{\partial z}\right)^{2}\right)\right. \\
& \left.+\left(\frac{\partial u}{\partial y}+\frac{\partial v}{\partial x}\right)^{2}+\left(\frac{\partial u}{\partial z}+\frac{\partial w}{\partial x}\right)^{2}+\left(\frac{\partial v}{\partial z}+\frac{\partial w}{\partial y}\right)^{2}\right]+\frac{\mu}{K T}\left(u^{2}+v^{2}+w^{2}\right)
\end{aligned}
$$

The first term on the right hand side of above equation is due to the heat transfer $\left(\dot{S}_{G H}^{\prime \prime \prime}\right)$ while the second and third terms are due to the viscous and Darcy dissipations $\left(\dot{S}_{G V}^{\prime \prime \prime}\right)$. It is well known that the viscous and Darcy dissipations are negligible in the first law of thermodynamics in many applications in clear fluid flow but it cannot be neglected in porous medium. The ratio $\dot{S}_{G H}^{\prime \prime \prime} / \dot{S}_{G V}^{\prime \prime \prime}$, becomes $\left(T_{R} / \Delta T_{R}\right) R_{I}$, where $R_{I}$ is the ratio of 
viscous dissipation to conduction in the first law with $T_{R}$ and $\Delta T_{R}$ indicating the characteristics scales, which, in the present reference, mention for an average temperature, $\left(T_{\text {top }}+T_{\text {bot }}\right) / 2$, and the temperature difference, $\left(T_{\text {bot }}-T_{\text {top }}\right)$, respectively. Hence, even if the dissipations are not negligible in the first law (which is the case of present study); it may be important in the second law due to the presence of the factor $\left(T_{R} / \Delta T_{R}\right)$. Our objective is to evaluate the cross sectional entropy generation, $\dot{S}_{G}^{\prime}[\mathrm{W} / \mathrm{m} \cdot \mathrm{K}]$, for fully developed mixed convection by integrating $\dot{S}^{\prime \prime \prime}$ over a cross section $A_{c}$; i.e.

$$
\dot{S}_{G}^{\prime}=\dot{S}_{G H, f d m c}^{\prime}+\dot{S}_{G V, f d m c}^{\prime}=\left\langle\dot{S}_{G H, f d m c}^{\prime \prime \prime}\right\rangle+\left\langle\dot{S}_{G V, f d m c}^{\prime \prime \prime}\right\rangle
$$

where $\langle F\rangle=\iint_{A_{c}} F \mathrm{~d} x \mathrm{~d} y$.

The subscript, $f d m c$, stands for the fully developed mixed convection. Since the temperature variations are small compared to the absolute temperature $T$, the factors, $k / T^{2}$ and $\mu / T$, in Equation (6) are assumed to be constant in performing the integrations.

For the rate of entropy generation due to the heat transfer, we obtain, after applying integration by parts as well as the adiabatic side wall conditions,

$$
\dot{S}_{G H, f d m c}^{\prime}=\frac{k}{T^{2}}\left\langle\left(\frac{\partial T}{\partial x}\right)^{2}+\left(\frac{\partial T}{\partial y}\right)^{2}\right\rangle=\frac{k}{T^{2}}\left[\left.\int_{0}^{2 W_{d}} T \frac{\partial T}{\partial y}\right|_{y=0} ^{H} \mathrm{~d} x-\left\langle T\left(\frac{\partial^{2} T}{\partial x^{2}}+\frac{\partial^{2} T}{\partial y^{2}}\right)\right\rangle .\right.
$$

The second term on the right hand side is zero as we apply conservation of energy, Equation (5), the hydrodynamic boundary conditions and integration by parts; i.e.

$$
\begin{aligned}
\left\langle T\left(\frac{\partial^{2} T}{\partial x^{2}}+\frac{\partial^{2} T}{\partial y^{2}}\right)\right\rangle & =\frac{1}{D_{T}}\left\langle T\left(u \frac{\partial T}{\partial x}+v \frac{\partial T}{\partial y}\right)\right\rangle=\frac{1}{2 D_{T}}\left\langle u \frac{\partial T^{2}}{\partial x}+v \frac{\partial T^{2}}{\partial y}\right\rangle \\
& =-\frac{1}{2 D_{T}}\left\langle T^{2}\left(\frac{\partial u}{\partial x}+\frac{\partial v}{\partial y}\right)\right\rangle=0 .
\end{aligned}
$$

Also by integrating conservation of energy, Equation (5), over a cross section it may be shown that heat transfer at the top boundary is balanced by heat transfer at the bottom boundary; i.e.

$$
\int_{0}^{2 W_{d}}\left(\frac{\partial T}{\partial y}\right)_{(y=H)} \mathrm{d} x=\int_{0}^{2 W_{d}}\left(\frac{\partial T}{\partial y}\right)_{(y=0)} \mathrm{d} x .
$$

Therefore,

$$
\dot{S}_{G H, f d m c}^{\prime}=\frac{k \Delta T}{T^{2}} \int_{0}^{2 W_{d}}\left(-\frac{\partial T}{\partial y}\right)_{(y=0)} \mathrm{d} x,
$$

with $\Delta T=T_{\text {bot }}-T_{\text {top }}$. In terms of a Rayleigh number, a Nusselt number and the mixing cup temperature, $T_{m}$, Equation (11) may be expressed as

$$
\overline{\dot{S}_{G H, f d m c}^{\prime}}=R a^{2} N u_{H}\left(1-\theta_{m}\right)
$$

where

$$
\begin{aligned}
& \overline{\dot{S}_{G H, f d m c}^{\prime}}=\frac{\dot{S}_{G H, f d m c}^{\prime}}{k A R / \gamma^{2}}, \quad \gamma=\frac{g \beta T H^{3}}{v D_{T}}, \quad A R=\frac{2 W_{d}}{H}, \quad R a=\frac{g \beta \Delta T H^{3}}{v D_{T}}, \quad \theta_{m}=\frac{T_{m}-T_{t o p}}{\Delta T}, \\
& N u_{H}=\frac{H \overline{\left.(-\partial T / \partial y)\right|_{y=0}}}{T_{b o t}-T_{m}}, \overline{\left.(-\partial T / \partial y)\right|_{y=0}}=\frac{1}{2 W_{d}} \int_{0}^{2 W_{d}} \frac{\partial T}{\partial y}(y=0) \mathrm{d} x
\end{aligned}
$$

with $\theta_{m, f d m c}$. Given $\Delta T$ and $A R$ for a specified fluid the entropy generation rate due to heat transfer is proportional to the Nusselt number.

The rate of entropy generation due to viscous and Darcy dissipations, upon application of the hydrodynamic conditions as well as continuity, becomes 


$$
\dot{S}_{G V, f d m c}^{\prime}=-\frac{\mu_{e}}{T}\left[u \nabla_{2}^{2} u+v \nabla_{2}^{2} v+w \nabla_{2}^{2} w\right]+\frac{\mu}{K T}\left(u^{2}+v^{2}+w^{2}\right),
$$

where $\nabla_{2}^{2}=\partial^{2} / \partial x^{2}+\partial / \partial y^{2}$. The first term on the right hand side of Equation (14), we may substitute conservation of momentum from Equation (2) to Equation (4), then repeated applications of integration by parts and the hydrodynamic boundary conditions yield,

$$
\begin{gathered}
-\frac{\mu}{T} u \nabla_{2}^{2} u=\frac{1}{T}\left\langle p^{\prime} \frac{\partial u}{\partial x}\right\rangle, \\
-\frac{\mu}{T} v \nabla_{2}^{2} v=\frac{1}{T}\left\langle p^{\prime} \frac{\partial u}{\partial x}\right\rangle+\frac{\rho g \beta}{T}\left\langle v\left(T-T_{R}\right)\right\rangle, \\
-\frac{\mu}{T} w \nabla_{2}^{2} w=\frac{1}{T}\left(-\frac{\mathrm{d} \bar{p}}{\mathrm{~d} z}\right)\langle w\rangle .
\end{gathered}
$$

Therefore,

$$
\dot{S}_{G V, f d m c}^{\prime}=\frac{1}{T}\left(-\frac{\mathrm{d} \bar{p}}{\mathrm{~d} z}\right)\langle w\rangle+\frac{\rho g \beta}{T}\left\langle v\left(T-T_{R}\right)\right\rangle .
$$

The first term on the right hand side is due to the forced convection (buoyancy induced flow). To represent the first term in Equation (17) in terms of non-dimensional parameters relevant to forced convection, we introduce

$$
f(\text { friction factor })=\frac{(-\partial \bar{p} / \partial z)}{\rho w_{m}^{2} / 2 D_{h}},
$$

where $w_{m}$ and $D_{h}$ are mean $z$-direction velocity and hydraulic diameter respectively, yielding

$$
\frac{1}{T}\left(-\frac{\mathrm{d} \bar{p}}{\mathrm{~d} z}\right)\langle w\rangle=\frac{k A R}{2 \gamma^{2}}\left(\frac{1+A R}{2 A R}\right)^{2} \gamma R a f P e E c .
$$

The rate of entropy generation due to the viscous dissipation associated with the buoyancy-induced flow in porous medium, on the other hand, may be expressed as

$$
\frac{\rho g \beta}{T}\left\langle v\left(T-T_{R}\right)\right\rangle=\frac{k A R}{\gamma^{2}} \Omega R a\langle\langle\bar{v} \theta\rangle\rangle,
$$

where $\langle\langle\bar{v} \theta\rangle\rangle=\int_{00}^{1} \int_{0}^{1}\left(v H / D_{T}\right)(\theta) \mathrm{d} \bar{x} \mathrm{~d} \bar{y}$ with $\bar{x}=x / 2 W_{d}, \bar{y}=y / H, \Omega=\rho\left(g \beta H^{2}\right)^{2} T / v k$.

Therefore,

$$
\dot{S}_{G V, f d m c}^{\prime}=\frac{1}{2}\left(\frac{1+A R}{2 A R}\right)^{2} \gamma R a f P e E c+\Omega R a\langle\langle\bar{v} \theta\rangle\rangle .
$$

The rate of non-dimensional entropy generation over a cross section for fully developed mixed convection in porous medium, $\overline{\dot{S}_{G, f d m c}^{\prime}}$, is

$$
\overline{\dot{S}_{G, f d m c}^{\prime}}=R a^{2} N u_{H}\left(1-\theta_{m}\right)+\frac{1}{2}\left(\frac{1+A R}{2 A R}\right)^{2} \gamma \operatorname{RafPeEc}+\Omega R a\langle\langle\bar{v} \theta\rangle\rangle .
$$

The right hand side of Equation (23) consists of contributions from heat transfer, cross-sectional (buoyancy-induced) flow and longitudinal main flow.

Now if Equation (5) includes the viscous as well as Darcy dissipations, i.e.

$$
\begin{aligned}
\rho c_{p}\left(u \frac{\partial T}{\partial x}+v \frac{\partial T}{\partial y}+w \frac{\partial T}{\partial z}\right)= & k\left(\frac{\partial^{2} T}{\partial x^{2}}+\frac{\partial^{2} T}{\partial y^{2}}+\frac{\partial^{2} T}{\partial z^{2}}\right)+\mu_{e}\left[2\left(\left(\frac{\partial u}{\partial x}\right)^{2}+\left(\frac{\partial v}{\partial y}\right)^{2}+\left(\frac{\partial w}{\partial z}\right)^{2}\right)\right. \\
& \left.+\left(\frac{\partial u}{\partial y}+\frac{\partial v}{\partial x}\right)^{2}+\left(\frac{\partial u}{\partial z}+\frac{\partial w}{\partial x}\right)^{2}+\left(\frac{\partial v}{\partial z}+\frac{\partial w}{\partial y}\right)^{2}\right]+\frac{\mu}{K}\left(u^{2}+v^{2}+w^{2}\right),
\end{aligned}
$$


then Equation. (23) becomes

$$
\overline{\dot{S}_{G, f d m c}^{\prime}}=R a^{2} N u_{H}\left(1-\theta_{m}\right)+2\left[\frac{1}{2}\left(\frac{1+A R}{2 A R}\right)^{2} \gamma R a f P e E c+\Omega R a\langle\langle\bar{v} \theta\rangle\rangle\right] .
$$

\section{Result and Discussions}

Entropy is a thermo-dynamical property that is a measure of the energy unavailable for useful work in a thermodynamic process, such as in energy conversion devices, engines, or machines, which can only be driven by convertible energy. When a substance is heated or cooled, there is a change in the entropy and has a theoretical entropy minimization (maximum efficiency) while converting the energy to useful work. From Equation (23) and Equation (25), we see that, if the thermal energy equation includes the viscous as well as Darcy dissipations then the second term (due to viscous dissipation) on the right hand side of the entropy generation rate is double and there is no change in first term (due to heat transfer).

\section{Conclusion}

This paper presents the analytical calculation for the non-dimensional entropy generation and the obtained result shows that there is no need to include the viscous and Darcy dissipations in the energy equation.

\section{Acknowledgements}

The author (SLY) would like to thank the Council of Scientific and Industrial Research, New Delhi, India, for financial support in the form of a Junior Research Fellowship.

\section{References}

[1] Brinkman, H.C. (1947) On the Permeability of Media Consisting of Closely Packed Porous Particles. Applied Scientific Research, A1, 81-86.

[2] Lundgren, T.S. (1972) Slow Flow through Stationary Random Beds and Suspensions of Spheres. Journal of Fluid Mechanics, 51, 273-299. http://dx.doi.org/10.1017/S002211207200120X

[3] Adler, P.M. and Mills, P.M. (1979) Motion and Rupture of a Porous Sphere in a Linear Flow Field. Journal of Rheology, 23, 25-37. http://dx.doi.org/10.1122/1.549514

[4] Kim, S. and Russell, W.B. (1985) Modelling in Porous Media by Renormalization of Stokes Equations. Journal of Fluid Mechanics, 154, 269-286. http://dx.doi.org/10.1017/S0022112085001525

[5] Koplik, J., Levine, H. and Zee, A. (1983) Viscosity Renormalization in the Brinkman Equation. Physics of Fluids, 26, 2864-2870. http://dx.doi.org/10.1063/1.864050

[6] Kolodziej, J.A. (1988) Influence of the Porosity of a Porous Medium on the Effective Viscosity in Brinkman's Filtration Equation. Acta Mechanica, 75, 241-254. http://dx.doi.org/10.1007/BF01174638

[7] Nield, D.A. and Bejan, A. (2013) Convection in Porous Media. Springer, New York. http://dx.doi.org/10.1007/978-1-4614-5541-7

[8] Breugem, W.P. (2007) The Effective Viscosity of Channel-Type Porous Medium. Physics of Fluids, 19, 1-16. http://dx.doi.org/10.1063/1.2792323

[9] Bejan, A. (2013) Convection Heat Transfer. 4th Edition, Wiley, New York. http://dx.doi.org/10.1002/9781118671627

[10] Bejan, A. (1982) Second Law Analysis in Heat Transfer and Thermal Design. Advances in Heat Transfer, 15, 1-58. http://dx.doi.org/10.1016/S0065-2717(08)70172-2

[11] Sahin, A.Z. (1998) A Second Law Comparison for Optimum Shape of Duct Subjected to Constant Wall Temperature and Laminar Flow. Heat and Mass Transfer, 33, 425-430. http://dx.doi.org/10.1007/s002310050210

[12] Narusawa, U. (2001) The Second Law Analysis of Mixed Convection in Rectangular Ducts. Heat and Mass Transfer, 37, 197-203. http://dx.doi.org/10.1007/s002310000173

[13] Mahmud, S. and Fraser, R.A. (2003) The Second Law Analysis in Fundamental Convective Heat Transfer Problems. International Journal of Thermal Sciences, 42, 177-186. http://dx.doi.org/10.1016/S1290-0729(02)00017-0

[14] Mohammad, A.A. (2003) Heat Transfer Enhancement in Heat Exchanger Fitted with Porous Media. Part, I.: Constant Wall Temperature. International Journal of Thermal Sciences, 42, 385-395. 
http://dx.doi.org/10.1016/S1290-0729(02)00039-X

[15] Sauoli, S. and Aiboud-Sauoli, S. (2004) Second Law Analysis of Laminar Falling Liquid Film along an Inclined Heated Plate. International Communication in Heat and Mass Transfer, 31, 879-886. http://dx.doi.org/10.1016/S0735-1933(04)00074-0

[16] Makinde, O.D. and Osalusi, E. (2005) Second Law Analysis of Laminar Flow in a Channel Field with Saturated Porous Media. Entropy, 7, 148-160. http://dx.doi.org/10.3390/e7020148

[17] Yadav, S.L. and Singh, A.K. (2014) Effects of Viscous and Darcy Dissipations on Entropy Generation Rate of Flow through a Horizontal Porous Channel. International Journal of Energy and Technology, 6, 1-7.

[18] Yadav, S.L. and Singh, A.K. (2016) Analysis of Entropy Generation in Annular Porous Duct. Transport in Porous Media, 111, 425-440. http://dx.doi.org/10.1007/s11242-015-0602-x

[19] Neale, G. and Nader, W. (1974) Practical Significance of Brinkman's Extension of Darcy's Law-Coupled Parallel Flows within a Channel and a Bounding Porous-Medium. The Canadian Journal of Chemical Engineering, 52, 475478. http://dx.doi.org/10.1002/cjce.5450520407 


\section{List of Symbol}

$A R$ aspect ratio, $2 W_{d} / H$

$c_{p}$ specific heat at constant pressure

$D_{h}$ hydraulic diameter

$E_{c}$ Eckert number, $w_{m}^{2} / c_{p} \Delta T$

$f$ friction factor, $(-\partial \bar{p} / \partial z) /\left(\rho w_{m}^{2} / 2 D_{h}\right)$

$g$ gravitational acceleration

$H$ height of the duct

$k$ thermal conductivity

$\underline{N u_{H}}$ average Nusselt number at the bottom based on $H, H \overline{\left.(-\partial T / \partial y)\right|_{y=0}} /\left(T_{b o t}-T_{m}\right)$ with

$\overline{(\partial T / \partial y)_{b o t}}$ being the temperature gradient at the bottom averaged over the width

$p$ pressure

$P e$ Peclet number, $w_{m} D_{h} / D_{T}$

Pr Prandtl number

$R a$ Rayleigh number, $g \beta \Delta T H^{3} / v D_{T}$

$\dot{S}_{G}^{\prime}$ cross sectional entropy generation

$\stackrel{\dot{S}_{G}^{\prime}}{\dot{S}_{G}^{\prime}} \dot{S}_{G}^{\prime} /\left(k A R / \gamma^{2}\right)$

$\dot{S}_{G}^{\prime \prime \prime} \quad$ local volumetric rate of entropy generation

$T_{m}$ mixing-cup temperature

$T_{b o t}$ temperature of the bottom boundary

$T_{\text {top }}$ temperature of the top boundary

$\Delta T \quad\left(T_{b o t}-T_{\text {top }}\right)$

$u$ velocity in $x$ (lateral) direction

$v$ velocity in $y$ (vertical) direction

$w$. velocity in $z$ (main flow) direction

$w_{m}$ mean velocity in $z$-direction

$W_{d}$ half width of a duct

$x$ transverse coordinate

$y$ vertical coordinate

$z$ longitudinal coordinate

\section{Greek Symbols}

$\gamma \quad g \beta T H^{3} / v D_{T}$

$\theta$ non-dimensional temperature $\left(T-T_{\text {top }}\right) /\left(T_{\text {bot }}-T_{\text {top }}\right)$

$\theta_{m}$ non-dimensional mixing-cup temperature

$\mu$ absolute viscosity

$\mu_{e}$ viscosity in presence of porous media

$v$ kinematic viscosity

$\rho$ density

$\Omega \rho\left(g \beta H^{2}\right)^{2} T / v k$

\section{Subscripts}

$f d$ fully-developed

$f_{c}$ forced convection

$H$ due to heat transfer

$m c$ mixed convection

$V$ due to viscous and Darcy dissipations 


\section{Submit or recommend next manuscript to SCIRP and we will provide best service for you:}

Accepting pre-submission inquiries through Email, Facebook, LinkedIn, Twitter, etc.

A wide selection of journals (inclusive of 9 subjects, more than 200 journals)

Providing 24-hour high-quality service

User-friendly online submission system

Fair and swift peer-review system

Efficient typesetting and proofreading procedure

Display of the result of downloads and visits, as well as the number of cited articles

Maximum dissemination of your research work

Submit your manuscript at: http://papersubmission.scirp.org/ 